\title{
STUDY OF HEAT FLUX GAGES USING SENSITIVITY ANALYSIS ${ }^{1}$
}

\author{
Kevin J. Dowding, Ben F. Blackwell, and Robert J. Cochran
}

Thermal Sciences Department 9113, M/S 0835

Sandia National Laboratories

Albuquerque, New Mexico, 87185-0835

\section{ABSTRACT}

The response and operation of a heat flux gage is studied using sensitivity analysis. Sensitivity analysis is the process by which one determines the sensitivity of a model output to changes in the model parameters. This process uses sensitivity coefficients that are defined as partial derivatives of field variables - e.g., temperature - with respect to model parameters - e.g., thermal properties and boundary conditions). Computing sensitivity coefficients, in addition to the response of a heat flux gage, aids in identifying model parameters that significantly impact the temperature response. A control volume, finite elementbased code is used to implement numerical sensitivity coefficient calculations, allowing general problems to be studied. Sensitivity coefficients are discussed for the well known Gardon gage.

\section{NOMENCLATURE}

$\mathcal{A} \quad$ area, $m^{2}$

C volumetric heat capacity, $J / m^{3} \mathrm{~K}$

$e^{\prime \prime \prime \prime} \quad$ volumetric source, $W / m^{3}$

$h \quad$ convection coefficient, $W / m^{2} K$

$k$ thermal conductivity, $W / m K$

$L \quad$ length of Gardon gage, $m$

$q^{\prime \prime} \quad$ heat flux, $W / m^{2}$

$\vec{q}$ heat flux vector, $W / m^{2}$

1. Sandia is a multiprogram laboratory operated by Sandia Corporation, a Lockheed Martin Company, for the United States Department of Energy under Contract DE-AC/04-94AL85000.

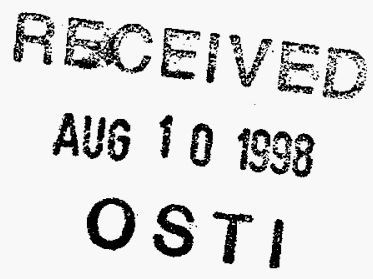

$q_{0}{ }^{\prime \prime} \quad$ boundary heat flux, $W / m^{2}$

$R \quad$ radius of Gardon gage, $m$

$T \quad$ temperature, $K$

$T_{i} \quad$ initial temperature, $K$

$T_{0} \quad$ temperature at center of Gardon gage, $K$

$T_{\beta} \quad$ scaled temperature sensitivity coefficient for parameter $\beta, K$

$T_{\infty} \quad$ convection temperature, $K$

$\nu \quad$ volume, $m^{3}$

$x, y, r \quad$ spatial coordinates

Greek:

$\alpha \quad$ thermal diffusivity, $\mathrm{m}^{2} / \mathrm{s}$

$\beta \quad$ parameter

$\delta$ thickness of Gardon gage, $m$

$\varepsilon \quad$ emissivity

$\sigma_{\beta} \quad$ standard deviation of parameter $\beta$

$\sigma_{T} \quad$ standard deviation of temperature, $K$

$\rho$ density, $\mathrm{kg} / \mathrm{m}^{3}$

\begin{tabular}{ll}
\multicolumn{2}{l}{ Subscripts } \\
$b$ & boundary \\
$B$ & base (copper) \\
$f$ & foil (constantan) \\
$w$ & wire (copper)
\end{tabular}

MASTER

DISTRLUTIOA OF THS DOCUMENT IS UMLABTED 


\section{DISCLAIMER}

This report was prepared as an account of work sponsored by an agency of the United States Government. Neither the United States Government nor any agency thereof, nor any of their employees, makes any warranty, express or implied, or assumes any legal liability or responsibility for the accuracy, completeness, or usefulness of any information, apparatus, product, or process disclosed, or represents that its use would not infringe privately owned rights. Reference herein to any specific commercial product, process, or service by trade name, trademark, manufacturer, or otherwise does not necessarily constitute or imply its endorsement, recommendation, or favoring by the United States Government or any agency thereof. The views and opinions of authors expressed herein do not necessarily state or reflect those of the United States Government or any agency thereof. 


\section{DISCLAIMER}

Portions of this document may be illegible in electronic image products. Images are produced from the best available original document. 


\section{INTRODUCTION}

Sensitivity analysis is an important and useful analysis tool. Sensitivity analysis means that the partial derivative of the state variable (temperature for thermal problems) with respect to physical model parameters (thermal conductivity, volumetric heat capacity, convection coefficient, emissivity, etc.) is calculated. This partial derivative is called a sensitivity coefficient. Valuable insight is gleaned from sensitivity coefficients. Sensitivity coefficient use and computation is discussed in this paper. In particular it is demonstrated how sensitivity coefficients can provide information concerning the effect of parameters on the response of heat flux gages. The insight from the sensitivity analysis can aid in gage design and improve our understanding of the gage's response. With a better understanding of the measured response, and more importantly what factors are influential on the response, more accurate data reduction can be achieved. Data reduction refers to the analysis relating the gage's measured response to the incident surface or absorbed heat flux.

Under certain circumstances data reduction for the heat flux gages involves an algebraic relationship between the measured response and heat flux. This relationship occurs because the response can be analytically calculated. When the assumptions for the simple relationship are not valid, inverse techniques may be needed. Inverse techniques allow a complex model, such as a finite element model, to be used in conjunction with measurements to estimate parameters or functions in the model that cannot be measured. In practice the heat flux gage predicts the heat flux at the surface of the gage from a measured temperature response. This problem is the classic inverse heat conduction problem (IHCP), Beck et al. (1985). The IHCP has added difficulty due to its illposed nature. Sensitivity coefficients are key to understanding how the measured response can be used to estimate the heat flux and possibly other parameters.

The importance of observing sensitivity coefficients has been widely advocated in parameter estimation by Beck; see Beck and Arnold (1977), Beck (1970), and Beck (1996). Other researchers have also used sensitivity coefficients to derive insight for parameter estimation; see Dowding et al. (1998), Marchall and Milos (1997), and Vozar and Sramkova (1997). Their use to designing an optimal parameter estimation experiment is discussed in Beck and Arnold (1977, Chapter 8); Taktak et al. (1993); and Emery and Fadale (1990, 1996, and 1997). Moffat (1985 and 1982) has suggested sensitivity coefficients be computed in analysis codes to quantify the experimental uncertainty associated with complex analyses. It is straightforward to compute experimental uncertainty and propagate error through an analysis code using perturbation methods when sensitivity coefficients are available.

Measuring the heat flux emanating from a radiative and/or convective source, such as a fire, is difficult. A common measurement approach uses a device that relates an easily measured response to the incident surface heat flux. A device that applies this approach is the circular foil, or Gardon gage, (Gardon, 1953). Gardon's original paper described the theory of operation for the Gardon gage. Since then, several investigations have studied the influence of heat loss modes initially neglected by Gardon; see Malone (1967), Kirchhoff (1972), Borell and Diller (1987), and Kuo and Kulkarni (1991). Additionally, Ash (1969) and Keltner and Wildin (1972) investigated the time response of the gage, which was initially assumed exponential in time by Gardon (1953).
Previously noted studies have demonstrated the influence of an effect - for example, heat losses by natural convection - on the response of the gage. To address the significance of an effect, the gage's response is often compared to the response neglecting the effect. Sensitivity coefficients provide a means to quantify the significance of an effect on the response but in reference to other physical model effects. This provides two insights. First, it allows physical effects in the model to be ranked according to importance. Second, it shows how dependent the response is on the parameters in the model. From a design standpoint it is essential to minimize the dependence on parameters that are least understood. When this is not feasible, efforts to better understand the parameters should be started. With the simplistic design of the Gardon gage, many insightful analytical models have been developed. The numerical solution studied in this paper permits a more general study of all the relevant physics. The addition of sensitivity coefficients to a numerical solution greatly enhances the value of the results.

In this paper sensitivity coefficients are used as an analysis tool to develop insight into the operation of heat flux gages. In the following section sensitivity coefficients and sensitivity equations are described. Sensitivity coefficients for a Gardon gage are presented and discussed in the subsequent section. Insight derived from sensitivity coefficients is described. A summary concludes the paper.

\section{SENSITIVITY COEFFICIENTS}

\section{Definition}

Although there are other dependent variable for which sensitivity is important, sensitivity coefficients related to temperature are studied in this paper. The sensitivity coefficient is the partial derivative of temperature with respect to a parameter, which is $\partial T / \partial \beta$ for a parameter $\beta$. Scaling allows sensitivity coefficients for different parameters to be compared. A scaled (sometimes called "modified") sensitivity coefficient is:

$$
T_{\beta} \equiv \beta \frac{\partial T}{\partial \beta} .
$$

Some papers represent the sensitivity coefficient for parameter $\beta$ with $X_{\beta}$. In this paper it is represented as $T_{\beta}$ because it has units of temperature, allowing sensitivity coefficient magnitudes for different parameters to be directly compared to temperature scales - e.g., magnitude of the transient temperature rise - within the problem.

\section{Sensitivity Coefficients for Complex Problems}

Complex problems with irregular geometry or multiple materials are generally solved with a numerical method. Several options exist to compute sensitivity coefficients with a numerical method. Blackwell et al. (1998) discuss the options and their implementation. The approach in this paper derives sensitivity equations by differentiating the describing equations and numerically solving the resulting equations. The details of the procedure are presented in Blackwell et al. (1998). Equations for temperature and sensitivity to parameters in the describing differential equation are discussed. The resulting equations are solved with a control volume finite element method with fully implicit time 
integration (Hogan et al., 1994); this procedure is not discussed in the paper.

Parameters in Differential Equation/Boundary Condition. The integral form of the energy equation for a heat conducting body is:

$$
\frac{\partial}{\partial t} \iiint_{V} C T d \mathcal{V}+\iint_{\mathcal{A}} \vec{q} \cdot d \overrightarrow{\mathcal{A}}=\iiint_{\mathcal{V}} \dot{e}^{\prime \prime \prime} d \mathcal{V}
$$

where the heat flux vector for isotropic thermal conductivity is given by Fourier's Law,

$$
\grave{q}=-k \nabla T \text {. }
$$

The boundary condition, assuming only a specified flux in this case, is

$$
\iint_{\mathfrak{A}_{b}} \vec{q} \cdot d \overrightarrow{\mathcal{A}}=\iint_{\mathfrak{A}_{b}}-(k \nabla T) \cdot d \overrightarrow{\mathcal{A}}=\iint_{\mathfrak{A}_{b}} q^{\prime \prime}{ }_{b} \hat{n} \cdot d \overrightarrow{\mathcal{A}},
$$

where $\mathcal{A}_{b}$ is along the boundary and

$$
q_{b}^{\prime \prime}=q_{o}^{\prime \prime}+h\left(T-T_{\infty}\right)
$$

In Eq. (5) the specified heat flux is comprised of constant and convective components.

Sensitivity equations are obtained by differentiating the energy equation, Eq. (2) through Eq. (5), with respect to a parameter and multiplying the result by the parameter. The sensitivity equation for isotropic conductivity is

$$
\frac{\partial}{\partial t} \iiint_{\mathcal{V}}\left(C T_{k}\right) d \mathscr{V}+\iint_{\mathscr{A}}\left(-k \nabla T_{k}\right) \cdot d \overrightarrow{\mathcal{A}}=\iint_{\mathscr{A}}[k \nabla T] \cdot d \overrightarrow{\mathcal{A}}
$$

with boundary condition

$$
\begin{gathered}
\iint_{\mathcal{A}_{b}}-\left(k \nabla T_{k}\right) \cdot d \overrightarrow{\mathcal{A}}=\iint_{\mathcal{A}_{b}}\left(k \frac{\partial q_{b}^{\prime \prime}}{\partial k}\right) \hat{n} \cdot d \overrightarrow{\mathcal{A}} \\
\left(k \frac{\partial q_{b}^{\prime \prime}}{\partial k}\right)=h T_{k} .
\end{gathered}
$$

In Eq. (6) to Eq. (8), the scaled sensitivity coefficient for thermal conductivity is

$$
T_{k} \equiv k \frac{\partial T}{\partial k}
$$

All thermal properties in Eq. (2) through Eq. (8) are assumed constant. Blackwell et al. (1998) discuss sensitivity equations for several other parameters and give a detailed derivation for sensitivity equations.

The right side term in Eq. (6), the sensitivity equation for conductivity, is the only nonhomogeneous term in the problem. It is the driving or forcing term for the sensitivity problem and contains the temperature. It is assumed that the temperature solution is already known, and the forcing term can be calculated. The forcing term is similar to a vol- umetric source/sink with magnitude equal to the negative of the heat flux. It is the gradient of heat flux that is important because large but equal values of heat flux across a control volume produce a zero net contribution. This dependence is more obvious after applying the divergence theorem to the right side of Eq. (6)

$$
\iint_{\mathfrak{A}}[k \nabla T] \cdot d \overrightarrow{\mathcal{A}}=\iint_{\mathcal{V}} \int(\nabla \cdot[k \nabla T]) d \mathcal{V}=-\iint_{\mathcal{V}} \int(\nabla \cdot \vec{q}) d \mathcal{V} .
$$

This equation demonstrates that the sensitivity coefficient for conductivity is indicative of heat flux gradients. A large heat flux gradient means the conductivity is restricting heat transfer by conduction and influencing the temperature. The sensitivity coefficient for conductivity is large in this case. The converse is not necessarily true, however. That is, a small sensitivity coefficient for conductivity does not necessarily mean its effect on temperature is insignificant. Rather it indicates that temperature is not sensitive to changes about the mean value of conductivity.

Temperature sensitivity does not provide complete information for the influence of conductivity. Heat flux sensitivity is especially pertinent in the operation of heat flux gages. Application of Fourier's Law gives heat flux sensitivity as

$$
\vec{q}_{\beta} \equiv \beta \frac{\partial \vec{q}}{\partial \beta}=\beta \frac{\partial}{\partial \beta}(-k \nabla T)=-\left[\left(\beta \frac{\partial k}{\partial \beta} \cdot \nabla T\right)+k \nabla T_{\beta}\right]
$$

Computing heat flux sensitivity requires postprocessing the temperature and temperature sensitivity. The first term on the right side is only nonzero for $\beta=k$ and is the heat flux for this case. For all other parameters the right side only contains the gradient of the sensitivity coefficients.

Parameters in the Boundary Condition. Sensitivity to parameters in the boundary conditions, such as a convection coefficient, convection temperature, etc., provide valuable insight as well. The sensitivity equation and boundary conditions for the convection coefficient are

$$
\begin{gathered}
\frac{\partial}{\partial t} \iint_{\mathcal{V}} \int_{\mathcal{A}}\left(C T_{h}\right) d \mathcal{V}+\iint_{\mathcal{A}}\left(-k \nabla T_{h}\right) \cdot d \overrightarrow{\mathcal{A}}=0 \\
\iint_{\mathcal{A}_{b}}-\left(k \nabla T_{h}\right) \cdot d \overrightarrow{\mathcal{A}}=\iint_{\mathcal{A}_{b}}\left(h \frac{\partial \dot{q}^{\prime \prime}}{\partial h}\right) \hat{n} \cdot d \overrightarrow{\mathcal{A}} \\
\left(h \frac{\partial \dot{q}_{b}^{\prime \prime}}{\partial h}\right)=h T_{h}+h\left(T-T_{\infty}\right) .
\end{gathered}
$$

In contrast to the sensitivity equations for thermal properties, the sensitivity equation for the convection coefficient, Eq. (12), is homogeneous. However, the boundary condition in Eq. (13) and Eq. (14) is not homogeneous. It contains an inhomogenous term (last term on the right side) that is identical to the boundary heat flux due to convection for temperature, Eq. (5). When heat flux due to convection is large, the sensitivity to convection coefficient is correspondingly large. Hence sensitivity to the convection coefficient shows the importance of boundary heat transfer due to convection. Similarly the sensitivity equations for a 


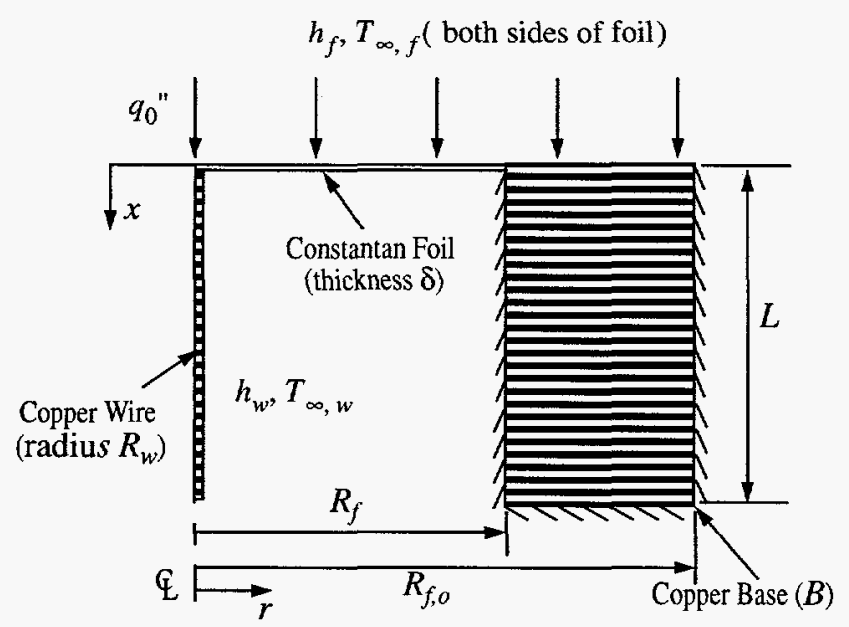

Figure 1. Model of Gardon gage

specified constant flux and for the emissivity would have driving terms identical to the boundary conditions for the temperature problem. These sensitivity coefficients would provide insight to the importance of heat transfer by the respective mode on the boundary. Consequently, sensitivity coefficients for different boundary condition parameters can be compared to rank the importance of heat transfer modes.

\section{RESULTS AND DISCUSSION}

Sensitivity coefficients are demonstrated by applying them to study a Gardon Gage. The impact of physical parameters describing the boundary conditions and thermal properties are studied to determine the influence of heat losses and identify important thermal properties.

A Gardon gage model is shown schematically in Figure 1. A circular constantan foil of radius $R_{f}$ has a copper wire attached to its center, and a copper base secures the outer radius of the foil. The copper wire and base are of length $L$. A constant heat flux is assumed across the top surface of the gage. Heat loss by natural convection from the foil surface and outer surface of the wire is also included. The surface of the copper base is assumed adiabatic. Heat loss due to radiation from the back side of the foil is neglected.

An ideal model neglects heat conduction to the center wire and convection from the foil surface and assumes the copper base maintains a constant temperature $\left(T_{B}\right)$ at the outer radius of the foil. With these assumptions only the foil is modeled, and the solution for the steady state temperature is (Kuo and Kulkarni, 1991)

$$
T(r)-T_{B}=\frac{q_{0}{ }^{\prime}}{4 k_{f} \delta}\left(R_{f}^{2}-r^{2}\right)
$$

Temperature difference between the center $(r=0)$ and outer radius of the constantan foil (or a proportional voltage) is measured. The analytical solution in Eq. (15) gives this response as

$$
T_{0}-T_{B}=\frac{q_{0}{ }^{\prime} R_{f}^{2}}{4 k_{f} \delta}
$$

Equation (15) is the analytical result that suggests a linear relationship is possible between the measured response and heat flux. However, the validity of this linear relationship depends on the gage design and operating conditions. Several researchers have shown conditions for which this model is not appropriate. Malone (1967) demonstrated the effect of convection from the foil surface and conductive losses to the center wire. The two-dimensional effects of the idealized case are studied by Kirchhoff (1972). The response of the gage to a step change in the heat flux or exposure to a convective environment is discussed by Ash (1969). Keltner and Wildin (1972) present the most detailed study of the gage response using a semianalytical method. Kuo and Kulkarni (1991) present corrections for a gage calibrated in a radiative environment (constant heat flux) but operated in a convective environment. Operation in a convective environment is discussed by Borell and Diller (1987). Grothus et al. (1983) present a detailed investigation. Several models are used to study the response, and parametric studies of physical gage dimensions and properties are shown. Primarily radiative conditions are studied, however.

The study of the Gardon gage in this paper is not intended to be a rigorous analysis of factors influencing the operation of the gage. Rather it demonstrates the use of sensitivity coefficients and the information obtained from them as a tool to understand factors influencing the gage. The following data from Keltner and Wildin (1972) and Kuo and Kulkarni (1991) are used to describe the gage studied:

$$
\begin{gathered}
\delta=R_{w}=0.00127 \mathrm{~cm} \\
R_{f}=0.311 \mathrm{~cm} \\
L=0.368 \mathrm{~cm} \quad R_{f, o}=0.411 \mathrm{~cm} \\
k_{f}=24.6(\mathrm{~W} / \mathrm{mK}) \quad k_{B}=k_{w}=397(\mathrm{~W} / \mathrm{mK}) \\
C_{f}=3.63 E 06\left(\mathrm{~J} / \mathrm{m}^{3} \mathrm{~K}\right) \quad C_{B}=C_{w}=3.645 \mathrm{E} 06\left(\mathrm{~J} / \mathrm{m}^{3} \mathrm{~K}\right) \\
q_{0}{ }^{\prime \prime}=1.0 \times 10^{4} \mathrm{~W} / \mathrm{m}^{2} .
\end{gathered}
$$

Heat losses due to convection from the foil and outer surface of the wire, assuming the same convection coefficient, $h_{f}=h_{w}$, are included. The coefficients are considered the same for simplicity. Numerical solutions are calculated using the physical gage data and discretized model in Figure 1. Approximately 360 finite elements are used $(2 \times 40$ in the foil, $60 \times 2$ in the wire, and $20 \times 8$ in the copper base, in the $x$ and $r$ direction, respectively).

\section{Influence of Convective Heat Losses}

The numerically computed temperature response $\left(T_{0}-T_{B}\right)$ with convective losses from the foil and wire is shown in Figure 2 for several values of the convection coefficient $\left(h=h_{f}=h_{w}\right)$. Temperature $\left(T_{B}\right)$ is taken as the value at the interface between the foil and base in the numerical model. The steady state response neglecting convective heat loss is approximately $78 \mathrm{~K}$. Heat loss reduces the response by 12 and 30 percent for convection coefficients of 10 and $30 \mathrm{~W} / \mathrm{m}^{2} \mathrm{~K}$, respectively. The heat flux predicted with a linear calibration (excluding heat losses) would have the same relative percent error. Clearly heat loss is signifi- 


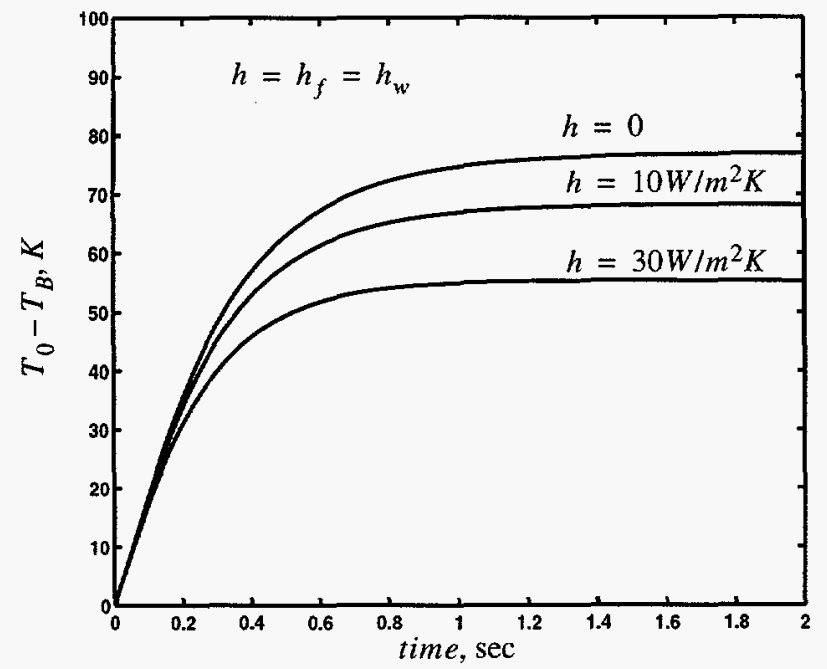

Figure 2. Gardon gage temperature response

cant. Sensitivity coefficients are studied next to determine what parameters influence the calculated response.

Sensitivity coefficients for the temperature difference $\left(T_{0}-T_{B}\right)$ are applicable for the Gardon gage. It can be shown that the sensitivity to a temperature difference is the difference of the sensitivity coefficients for the individual temperatures. It is noted that the sensitivity of temperature at the outer radius of the foil $\left(T_{B}\right)$ is approximately zero. Because temperature is nearly constant there, its value is insensitive to model parameters. Temperature sensitivity is exactly zero at a surface where temperature is prescribed.

Sensitivity coefficients are separated into two categories: those related to the boundary conditions and those related to thermal properties. Dependence on the thermal properties can be eliminated with a careful calibration. However, the gage must be operated within the calibration bounds, which typically neglect convective heat loss.

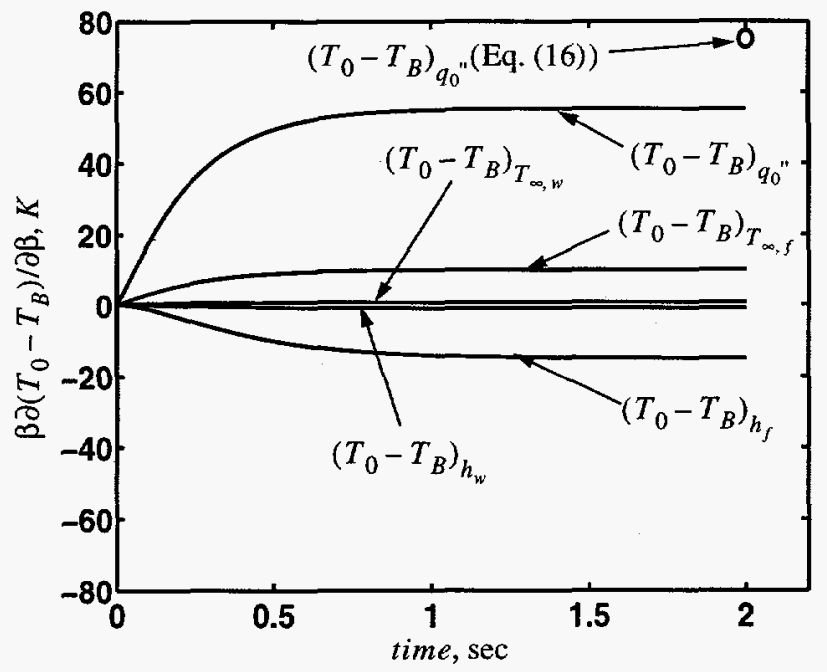

Figure 3. Sensitivity of Gardon gage temperature response to boundary condition parameters for $h=30 \mathrm{~W} / \mathrm{m}^{2} \mathrm{~K}$
Sensitivity to convection coefficients, ambient temperatures, and the applied heat flux is shown in Figure 3 for $h=30 \mathrm{~W} / \mathrm{m}^{2} \mathrm{~K}$. The most influential parameter on the response is the applied heat flux $q_{0}{ }^{\prime \prime}$. Its value closely approximates the temperature response as seen in Figure 2. If the ideal model is applicable, Eq. (16), the scaled sensitivity to the applied heat flux is identical to the steady state ideal temperature response. This value is shown on the plot for comparison. By comparing the sensitivity to the applied heat flux with and without convective losses at steady state, the gage is shown to be less sensitive to the applied flux when there are convective losses.

Equal magnitudes are assumed for the convection coefficients describing heat losses from the foil and wire surfaces. However, sensitivity to the convection coefficients is markedly different. Sensitivity to the convection coefficient and ambient temperature for the foil $\left(h_{f}\right.$ and $\left.\mathrm{T}_{\infty, f}\right)$ are more than an order of magnitude larger than those for the wire $\left(h_{w}\right.$ and $\mathrm{T}_{\infty, w}$ ). Recall that the sensitivity equation for parameters in boundary conditions showed that these sensitivity coefficients are indicative of the importance of heat losses. The magnitudes indicate that the gage's response is influenced more by losses from the foil than by losses from the wire. The foil has a significantly larger surface area; thus losses from it are more important.

Sensitivities for the parameters describing losses from the foil are further discussed. Sensitivity to the convection coefficient $\left(h_{f}\right)$ is about 80 percent larger than sensitivity to the convection temperature $\left(T_{\infty, f}\right)$. It is more important to know the convection coefficient than the ambient temperature. The coefficients have different signs; sensitivity to $h_{f}$ is negative indicating an increase in this parameter results in an decrease in the temperature; sensitivity to $T_{\infty, f}$ is positive indicating an increase in this parameter results in a increase in the temperature. This is not to imply that the two effects may cancel each other.

Sensitivity to the thermal properties, thermal conductivity and volumetric heat capacity, of the foil and wire are shown in Figure 4. The properties of the foil have the largest sensitivity coefficients. Sensitivity

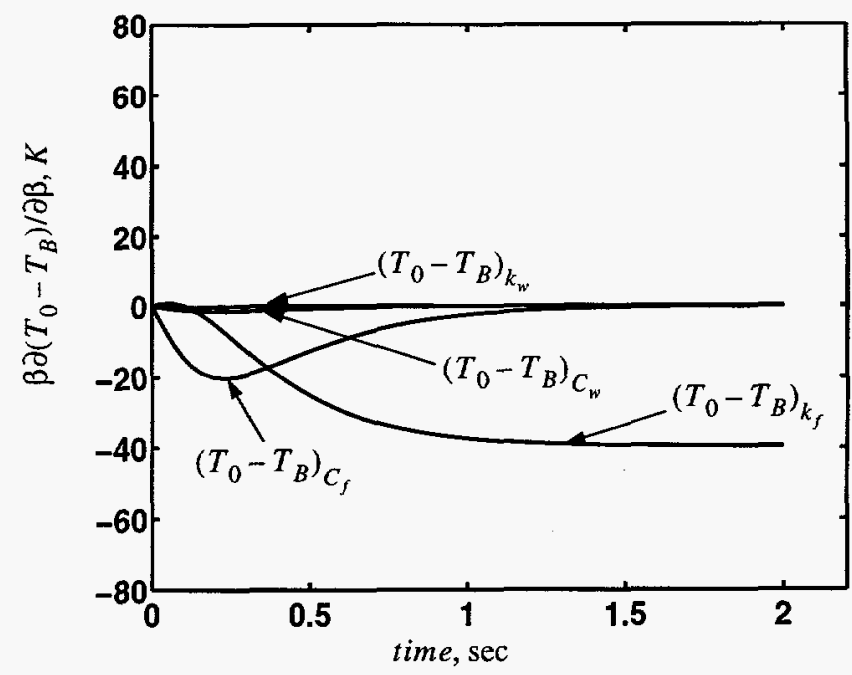

Figure 4. Sensitivity of Gardon gage temperature response to thermal properties for $h=30 \mathrm{~W} / \mathrm{m}^{2} \mathrm{~K}$ 
to the conductivity of the foil is maximum at steady state. Sensitivity to the heat capacity of the foil is maximum at approximately 0.25 seconds and decreases to zero at steady state. Thermal properties of the wire have small sensitivities. Small sensitivity indicates that the wire properties are comparably less significant on the response of the gage. It does not necessarily mean the wire can be neglected in the model. Sensitivity coefficients are computed for mean values of the parameters. A small sensitivity coefficient indicates that changes in the parameter, for the mean value of the parameters, have a small influence on the temperature. Although changes in the thermal properties of the wire are not significant, there could be appreciable conduction down the wire. When insensitivity to the thermal properties is coupled with insensitivity to the convective losses from the wire, the overall effect of the wire is deemed small.

Sensitivity analysis results are distilled to provide design/analysis information. Observations from the sensitivity analysis include the following:

1. The general insensitivity associated with the center wire suggest it can be excluded from the model with little effect on the response. See $T_{h_{w}}, T_{T_{\infty, w}}$ in Figure 3 and $T_{k_{w}}, T_{C_{w}}$ in Figure 4.

2. When the magnitude of the sensitivity coefficients for the heat losses from the foil $\left(h_{f}\right.$ and $\left.\mathrm{T}_{\infty, f}\right)$ are compared to that for $q_{0}$ " in Figure 3, it is concluded that neglecting heat losses from the foil is not appropriate. The scaled sensitivity to parameters describing heat losses from the foil have magnitudes in the range $10-15^{\circ} \mathrm{C}$, which are 15-25 percent of sensitivity to the applied heat flux.

3. Knowledge of the convection coefficient from the foil $\left(h_{f}\right)$ is more important than the convection temperature $\left(\mathbf{T}_{\infty, f}\right)$. See magnitudes in Figure 3.

4. Time response of the gage is most influenced by the volumetric heat capacity of the foil. Time response is typically taken to be the time to reach 63 percent of a step response. For the gage studied, the predicted time response is approximately $0.25 \mathrm{sec}$, which is near the time of maximum sensitivity to the volumetric heat capacity of the foil in Figure 4.

It is clear in Figure 2 that the response of the gage is impacted by the heat losses from the foil. A separate, but related, question is how much impact would knowledge of the thermal properties and heat loss coefficients have on the calculated response. The question is important for understanding how sensitive the calculated results are to specified physical model parameters. This study assumes that the applied heat flux $q_{0} "$ is known. Consequently, errors are assumed in all model parameters except for $q_{0} "$.

Uncertainty in the calculated response because of input uncertainties can be computed using perturbation methods. Input uncertainties are imprecise knowledge of thermal properties, convection coefficients, etc. It can be shown that for first order perturbation methods (Fadale, 1993), the uncertainty in the response is related to the input uncertainties as

$$
\sigma_{T}^{2}=\sum_{i=1}^{p}\left(T_{\beta_{i}} \frac{\sigma_{\beta_{i}}}{\beta_{i}}\right)^{2},
$$

where $\sigma_{T}$ is the estimated standard deviation of the temperature based on contributions of individual parameters. The contribution for each

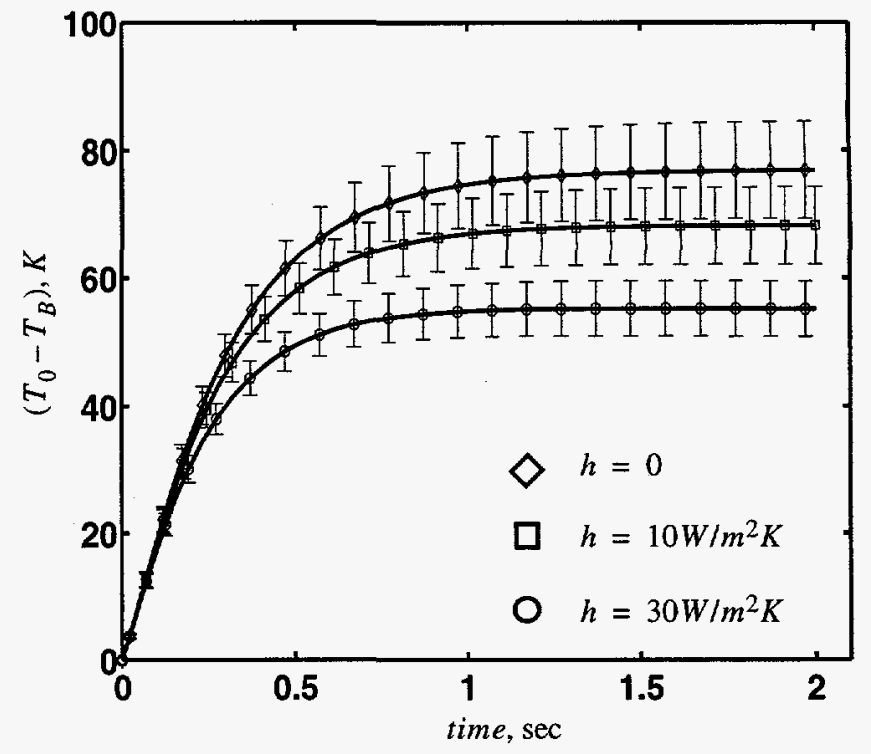

Figure 5. Uncertainty in temperature response of Gardon gage due to $10 \%$ uncertainty in thermal properties and heat loss parameters

parameter $\left(\beta_{i}\right)$ is the sensitivity coefficient $\left(T_{\beta_{i}}\right)$ multiplied by the normalized standard deviation for the parameter $\left(\sigma_{\beta_{i}} / \beta_{i}\right)$.

The uncertainty, plotted as $\pm \sigma_{T}$ error bars on the gage response, is shown in Figure 5. The uncertainty is computed by assuming a 10 percent normalized standard deviation in the thermal properties and boundary conditions. The uncertainty in the gage response becomes smaller as the convection coefficient increases. This trend is due to the sensitivity to the foil thermal properties. They are the dominant sensitivity coefficients, and hence have the most influence on the uncertainty. As the convection coefficient increases, these sensitivity coefficients decrease in magnitude, resulting in a decrease in the total uncertainty. The relative uncertainty, which is normalized by the steady state temperature, also decreases with increasing convection. The relative uncertainty is 10 percent for $h=0$ and decreases to 9 and 8 percent for convection coefficients of 10 and $30 \mathrm{~W} / \mathrm{m}^{2} \mathrm{~K}$, respectively.

\section{Calibration Data}

Calibration relates the measured response to the applied heat flux. Figure 6 shows calculated calibration curves for an ideal gage and gage that includes convective losses $\left(h=30 \mathrm{~W} / \mathrm{m}^{2}\right)$. Uncertainty bounds $\left( \pm \sigma_{T}\right)$ are shown (dashed lines) for the calibration including convective losses. Uncertainty is calculated assuming a $10 \%$ relative input uncertainty in thermal properties and convection coefficients and convection temperatures. For a given heat flux, convective losses reduce the response of the gage compared to the ideal model. The model with convective losses shows a greater deviation from the ideal model at larger heat fluxes (or temperature response). Borell and Diller (1987) demonstrate similar trends for an experimental calibration in a convective environment.

Typically a gage is calibrated by measuring its response to a known (incident) heat flux. For the assumptions discussed previously, a 


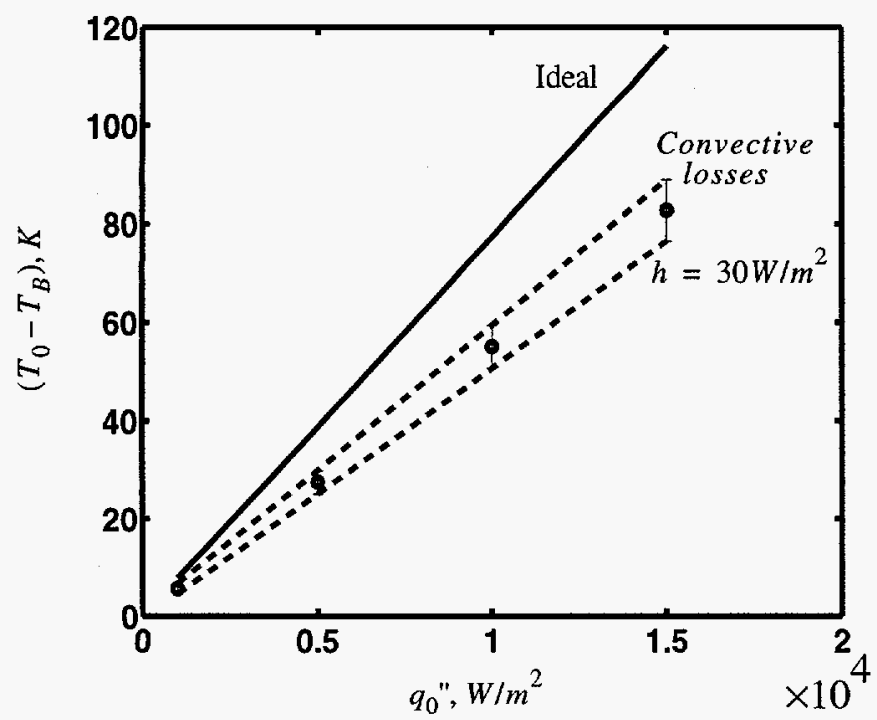

Figure 6. Calibration curves for Gardon gage

linear relationship between the response and heat flux is suggested, Eq. (16). However, such a calibration procedure does not check, nor verify, that the (assumed) model is correct. Trends in Figure 6 indicate that a model with convective losses has approximately a linear calibration. But, the calibration is quite different from the ideal gage when convective losses are included. A calibration done without studying the model may miss this difference. For the conditions used, the data suggests that convective losses are needed in the model to accurately describe its calibration.

Developing a model of the gage requires thermal properties and boundary conditions, which may not be accurately known. It is for this situation that the sensitivity coefficients and uncertainty bounds on the response provide additional insight. Knowing how sensitive the calculated response is to changes in the specified properties and boundary conditions is paramount to understanding the applicability of the solution. Uncertainty bounds help to quantify the influence of input uncertainty on the solution. Uncertainty in the response with convective losses in Figure 6 shows that as the heat flux (or temperature response) increases, the uncertainty in the predicted response increases. Therefore, the input uncertainty is more important at larger heat fluxes.

By experimentally calibrating the gage, influences due to thermal properties and other factors can admittedly be minimized or eliminated. Convection losses are not as easily handled. We feel that investigating the model and its associated sensitivity/uncertainty complements the experimental calibration. It demonstrates the adequacy of the ideal model, relative to a more complex model, without costly experimentation. Furthermore, in situations that require a complex model, inverse techniques could be used in conjunction with the model to estimate parameters, such as a convection coefficient. Such a procedure, though more difficult to analyze, could provide more accurate calibration curves. Calibration and operation of the gage in a convective environment is considerably more complex. Calibrations that are developed may be quite restricted in their applicability. Sensitivity studies help us understand factors that influence the applicability.

A final comment about the uncertainty is given. Figure 6 shows the uncertainty on the predicted response for a specified heat flux.
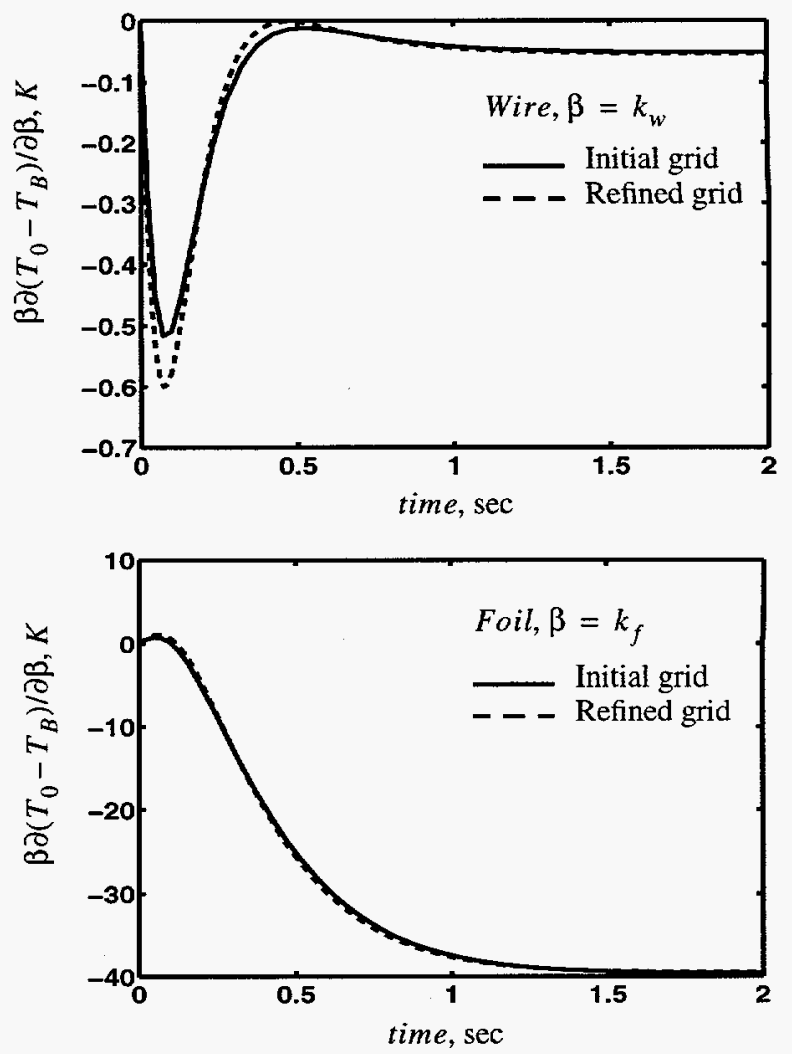

Figure 7. Sensitivity coefficients for the thermal conductivity, comparing coefficients after refining the grid

Relating this information to the uncertainty in the heat flux for a specified temperature response is currently under investigation. The uncertainty in the heat flux is more useful in practice.

\section{Grid Sensitivity}

Grid resolution issues are addressed by doubling the number of finite elements and reducing the time step by a factor of four. Calculated response for the refined mesh changes a maximum of 0.5 percent, relative to the steady state response.

A grid-converged solution for the temperature does not ensure the same for sensitivity coefficients. Experience has shown that sensitivity coefficients have regions of steep gradients that do not coincide with those for temperature. Furthermore, there may be variation between the grids required for different sensitivity coefficients. Consider the sensitivity coefficients for the thermal conductivities of the foil and wire shown in Figure 7. Sensitivity to the foil conductivity changes less than 1 percent (relative to the maximum) after refining the grid in the bottom plot. However, in the top plot the sensitivity to the wire properties changes over 15 percent. The magnitude of the sensitivity to wire conductivity is small in comparison to that for the foil. Hence although we may not have a grid-insensitive solution for $T_{k_{w}}$, with its small magnitude it is of little consequence. Sensitivity coefficients of appreciable size had less than a 1 percent relative change for the refined grid. 


\section{SUMMARY}

Sensitivity coefficients indicate the dependence of a calculated response on model parameters. Knowing the model parameters, such as thermal properties and boundary conditions, that the response is sensitive to gives important design/analysis information. Model parameters with large sensitivity coefficients can be further investigated to increase the model's fidelity. The importance of effects, such as convective losses, can be identified as well.

Sensitivity coefficients were used to study a heat flux gage. It was shown that the convective heat loss from the surface of a Gardon gage can impact the calculated response. The model of the gage included the center wire and convection from the foil surface. The sensitivity coefficients showed that the effect of the center wire was minimal. Sensitivity coefficients further showed that the uncertainty in the calculated response of the gage is impacted more by the properties of the foil than convective losses from the surface. In addition, a predicted calibration plot demonstrated that uncertainty grew as the temperature response increased. These conclusions were based on the mean parameters of the gage. If deviations from the mean are large, then the perturbation analysis should be repeated for a new set of mean parameters.

\section{ACKNOWLEDGEMENTS}

The authors would like to acknowledge Professor J. V. Beck of Michigan State University for having introduced us (BFB and KJD) to the useful concept of sensitivity coefficients.

\section{REFERENCES}

Ash, R. L., 1969, "Response Characteristics of Thin Foil Heat Flux Sensors," AIAA Journal, Vol. 7, No. 12, pp. 2332-2335.

Beck, J. V., 1996, "Parameter Estimation Concepts and Modeling: Flash Diffusivity Application," Proceeding of the Second International Conference on Inverse Problems in Engineering: Theory and Practice, eds. D. Delaunay, K. Woodbury, and M. Raynaud, 9-14 June 1996, LeCroisic, France, ASME Engineering Foundation.

Beck, J. V., Cole, K., Haji-Sheikh, A., and Litkouhi, B., 1992, Heat Conduction Using Green's Functions, Hemisphere, New York.

Beck, J. V., Blackwell, B., and C. R., St. Clair, 1985, Inverse Heat Conduction, Wiley, New York.

Beck, J. V., and Arnold, K. J., 1977, Parameter Estimation, Wiley, New York.

Beck, J. V., 1970, "Sensitivity Coefficients Utilized in Nonlinear Estimation With Small Parameters in a Heat Transfer Problem," Journal of Basic Engineering, June 1970, pp. 215-222.

Borell, G. J., and Diller, T. E., 1987, "A Convection Calibration Method for Local Heat Flux Gages," ASME Journal of Heat Transfer, Vol. 109, pp. 83-88.

Blackwell, B. F., Cochran, R. J., and Dowding, K. J., 1998, "Development and Implementation of Sensitivity Coefficient Equations For Heat Conduction Problems," ASME proceedings of the 7th AIAA/ ASME Joint Thermophysics and Heat Transfer Conference, Vol. 2, eds. A. Emery et al., ASME-HTD-357-2, pp. 303-316.

Dowding, K. J., Blackwell, B. F., and Cochran, R. J., 1998, "Application of Sensitivity Coefficients for Heat Conduction Problems," ASME proceedings of the 7th AIAA/ASME Joint Thermophysics and Heat Transfer Conference, Vol. 2, eds. A. Emery et al., ASMEHTD-357-2, pp. 317-327.
Emery, A. F., and Fadale, T. D., 1990, "Stochastic Analysis of Uncertainties in Emissivity and Conductivity by Finite Element," paper 90-WA/HT-11, presented at Winter Annual Meeting Dallas, TX, November 1990.

Emery, A. F., and Fadale, T. D., 1996, "Design of Experiments Using Uncertainty Information," ASME Joumal of Heat Transfer, Vol. 118 , pp. 532-538.

Emery, A. F., and Fadale, T. D., 1997, "Designing Thermal Systems with Uncertainty Properties using Finite Element/Volume Methods," Proceeding of the 32nd National Heat Transfer Conference, Vol. 2, eds. G. S. Dulikravich and K. A. Woodbury, Baltimore, MD, HTDVol 340, pp. 75-81.

Fadale, T. D., 1993, "Uncertainty Analysis using Stochastic Finite Elements," Ph.D. dissertation, University of Washington, Seattle, WA.

Gardon, R. G., 1953, "An Instrument for the Direct Measurement of Intense Thermal Radiation," Review of Scientific Instruments, Vol. 24, No. 5, pp. 366-370.

Grothus, G. A., Mulholland, G. P, Hills, R. G., and, Marshall, B. W., 1983, "The transient Response of Circular Foil Heat Flux Gages," report SAND83-0263, Sandia National Laboratories, Albuquerque, NM.

Hogan, R. E., Blackwell, B. F., and Cochran, R. J., 1994, "Numerical Solution of Two-Dimensional Ablation Problems Using the Finite Control Volume Method with Unstructured Grids," paper AIAA 942085, 6th AIAA/ASME Joint Thermophysics and Heat Transfer, Colorado Springs, CO, June 20-23, 1994.

Keltner, N. R., and Wildin, M. W., 1975, "Transient Response of Circular Foil Heat Flux Gages to Radiative Fluxes," Review of Scientific Instruments, Vol. 46, No. 5, pp. 1161-1166.

Kirchhoff, R. H., 1972, "Response of Finite-Thickness Gardon Heat-Flux Sensors," ASME Journal of Heat Transfer, Vol. 94, pp. 244 245.

Kuo, C. H., and Kulkarni, A. K., 1991, "Analysis of Heat Flux Measurements by Circular Foil Gages in a Mixed convection/Radiation Environment," ASME Journal of Heat Transfer, Vol. 113, pp. 1037 1040.

Malone, E. W., 1968, "Design and Calibration of Thin-Foil Heat Flux Sensors," ISA Transactions, Vol. 7, pp. 175-179.

Fadale, T. D., 1993, "Uncertainty Analysis using Finite Elements," Ph.D. Thesis, University of Washington.

Marschall, J., and Milos, F., 1997, "Simultaneous Estimation of Sensor Locations During Thermal Property Parameter Estimation," Proceeding of the 32nd National Heat Transfer Conference, Vol. 10, eds. R. Clarksean et. al, Baltimore, MD, HTD-Vol 348, pp. 79-89.

Moffat, R. J., 1985, "Using Uncertainty Analysis in the Planning of an Experiment," Journal of Fluids Engineering, Vol. 107, pp. 173182.

Moffat, R. J., 1982, "Contributions to the Theory of Single-Sample Uncertainty Analysis," Journal of Fluids Engineering, Vol. 104, pp. 250-260.

Taktak, R., Beck, J.V., and Scott, E., 1993, "Optimal Experimental Design for Estimating Thermal Properties of Composite Materials," International Journal of Heat Mass Transfer, Vol. 36, No. 12, pp. 2977 2986.

Vozar, L., and Sramkova, T, 1997, "Two data Reduction Methods for Evaluation of Thermal Diffusivity from Step-Heating Measurements," International Journal of Heat and Mass Transfer, Vol 40, No. 7, pp. 1647-1655. 